\title{
Numerical simulation of breast reduction with a new knitting condition
}

\author{
D. Lopes ${ }^{1}$, S. Clain ${ }^{2}$, R.M.S. Pereira ${ }^{2,3}$, G.J. Machado ${ }^{2}$, G. Smirnov ${ }^{3}$, I. Vasilevskiy ${ }^{3}$ \\ ${ }^{1}$ Department of Informatics, University of Minho, Campus of Gualtar, 4710-057 Braga, Portugal \\ ${ }^{2}$ Centre of Mathematics, University of Minho, Campus of Gualtar, 4710-057 Braga, Portugal \\ ${ }^{3}$ Centre of Physics, University of Minho, Campus of Gualtar, 4710-057 Braga, Portugal
}

\begin{abstract}
SUMMARY
Breast reduction is one of the most common procedures in breast surgery. The aim of this work is to develop a computational model allowing one to forecast the final breast geometry according to the incision marking parameters. This model can be used in surgery simulators that provide preoperative planning and training, allowing the study of the errors origin in breast reduction. From the mathematical point of view this is a problem of calculus of variations with unusual boundary conditions, known as knitting conditions. The breast tissue is considered as a hyperelastic material, discretized with three-dimensional finite elements for the body whereas the skin is modelled with two-dimensional finite elements on the curved surface. Although the model is of low precision, we show that it is sufficient for a satisfactory analysis of errors frequently done during breast reduction surgery and allows to understand how to avoid or correct them.
\end{abstract}

KEY WORDS: Breast surgery, numerical simulation, knitting conditions

\section{INTRODUCTION}

Breast reduction surgery is a procedure used to remove excess fat, tissue, and skin from the breasts in order to achieve a breast size in proportion with the body and to alleviate the discomfort associated with overly weight breast. The surgeon first makes one or more cuts and then the excess tissue and skin are removed while the nipple and areola are repositioned. At last, the skin is closed with stitches to initiate the natural suturing process. To help surgeons to take a better informed decision, medical numerical simulation enables to carry out virtual surgery operations to visualize the post-operated breast and to analyse several scenarios. The two main areas of the breast modelling applications are aesthetic surgery [1,2] and medical imaging analysis [3].

Although the number of works on the breast soft tissue modelling has increased significantly during the two last decades $[2,3,4,5,6]$, the development of an adequate breast model still continues to be a challenging problem. The use of nonlinear models to simulate the skin, the muscles, and the tissues is well-developed in the biomechanics context $[7,8,9]$ while the finite element method is a popular technique for the discretization $[10,11,12]$ using alternatively the weak formulation or the minimization framework [13, 14, 15]. The numerical simulation of this type of surgery gives rise to a very specific problem where one has to prescribe suturing conditions in order to provide realistic breast shape. Indeed, the junction of the two incision interfaces is complex: the interfaces are placed in contact while the border (skin) is sewn. From a numerical point of view, a technical difficulty derives from the non-matching of the left and right surface meshes

\footnotetext{
${ }^{*}$ Correspondence to: S. Clain, Centre of Mathematics, University of Minho, Campus of Gualtar, 4710-057 Braga, Portugal. E-mail: clain@math.uminho.pt
} 
which requires the development of a new numerical suturing algorithm. In [16] it was proposed a mathematical model and a numerical scheme to simulate the suturing after two typical incisions in the breast reduction context: a symmetric frontal cut and a rear cut. In this work we generalize the model in order to consider an asymmetric frontal cut.

From the mathematical point of view, the problem is formulated as a problem of calculus variations with unusual knitting conditions. The breast tissue is considered as a hyperelastic material and, although most of soft tissues are incompressible, we consider the breast as a compressible NeoHookean material as proposed in $[2,17]$. The breast tissue is modelled using three-dimensional elements while the skin is modelled using two-dimensional elements since its elastic properties are very different from those of the breast gland and fat tissue. Moreover, we include in the model the Chassaignac space since it is of great importance to simulate the breast submitted to the gravitational force.

The paper is organized as follows. We present in section 2 the mathematical formulation of the the suturing problem while in Section 3 we detail the numerical algorithms. The breast model in the gravitational field is given in Section 4 while its respective numerical scheme is designed in Section 5. Numerical tests are given in the sixth section where we present three typical configurations and in last section we present the conclusions of this work.

\section{MODELLING}

Suturing process model is based on two main ingredients: a nonlinear elasticity model of the breast and suturing conditions to perform the junction of the two interfaces. The last point is far from trivial since, in practice, the surgeon sutures the border of the interfaces between themselves while the inner parts of the interface only are in contact. Hence the boundary conditions should carefully be prescribed to correctly model the surgery.

\subsection{Geometry}

In order to mathematically model the suturing problem, we denote by $\Omega_{\text {cut }}$ the domain which corresponds to the incised breast and prescribe the conditions to perform the suturing. Since the suturing process is very complex from a geometrical point of view, one has to carefully define the interfaces of the breast and its parametrization. In short, the breast is considered as a spherical cap where we perform two cuts. We plot in Figure 1 three technical views with the parameters given in Table I.

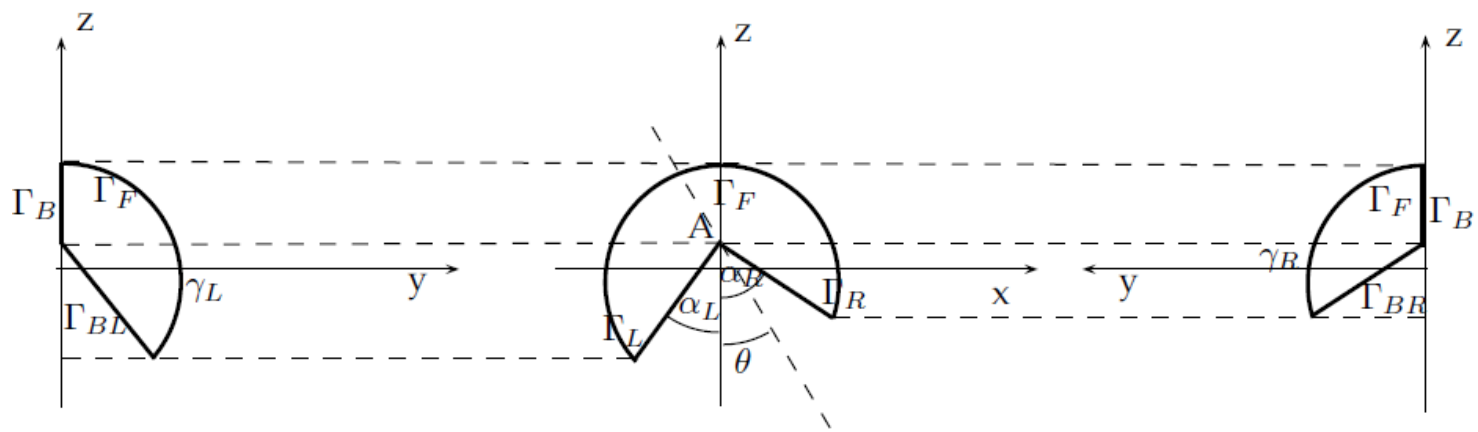

Figure 1. Scheme of breast reduction surgery: the middle panel is the frontal view while the left and right panels report the parameters for the left and right parts of the breast.

We use the following convention: $\Gamma$ represents a bi-dimensional surface while $\gamma$ corresponds to a generic one-dimensional curve. To describe the suturing problem, we introduce the following set of boundaries we display in Figure 2 while Table I gives an overview of the notations.

The breast is partially covered with a skin characterized by the boundary surface $\Gamma_{\mathrm{F}}$. The frontal 
Table I. List of symbols and notations.

$\begin{array}{ll}\Gamma_{\mathrm{B}} & \text { back side of the breast that is fixed to the chest } \\ \Gamma_{\mathrm{F}} & \text { surface associated to the skin of the breast } \\ \Gamma_{\mathrm{L}}, \Gamma_{\mathrm{R}} & \text { left and right cuts performed during the frontal incision } \\ \Gamma_{\mathrm{BL}}, \Gamma_{\mathrm{BR}} & \text { left and right cuts performed during the rear incision } \\ A & \text { point on the front of the breast where the first incision starts } \\ \gamma_{0}=\Gamma_{\mathrm{L}} \cap \Gamma_{\mathrm{R}} & \text { line between point } A \text { and the chest } \\ \gamma_{\mathrm{L}}=\Gamma_{\mathrm{F}} \cap \Gamma_{\mathrm{L}} & \text { frontal left suturing line } \\ \gamma_{\mathrm{R}}=\Gamma_{\mathrm{F}} \cap \Gamma_{\mathrm{R}} & \text { frontal right suturing line } \\ \gamma_{\mathrm{BL}}=\Gamma_{\mathrm{F}} \cap \Gamma_{\mathrm{BL}} & \text { backward left suturing line } \\ \gamma_{\mathrm{BR}}=\Gamma_{\mathrm{F}} \cap \Gamma_{\mathrm{BR}} & \text { backward right suturing line } \\ \theta & \text { angle of the suturing plane } \\ \alpha_{\mathrm{L}}, \alpha_{\mathrm{R}} & \text { left and right angles of the frontal incision }\end{array}$

incision is performed between the two interfaces denoted by $\Gamma_{\mathrm{L}}$ and $\Gamma_{\mathrm{R}}$ respectively sharing the line $\gamma_{0}=\Gamma_{\mathrm{L}} \cap \Gamma_{\mathrm{R}}$ passing at point $A$, orthogonal to the trunk body. A second rear incision is performed between the breast and the trunk such that a portion of the breast remains in contact with the body via the interface $\Gamma_{\mathrm{B}}$ while $\Gamma_{\mathrm{BL}}$ and $\Gamma_{\mathrm{BR}}$ are the left and right surfaces respectively deriving from rear incisions. Boundary lines $\gamma_{\mathrm{L}}$ and $\gamma_{\mathrm{R}}$ in red in Figure 2 correspond to the suturing line that the surgeon will join while $\gamma_{\mathrm{BL}}$ and $\gamma_{\mathrm{BR}}$ are the rear incision lines. Notice that one has $\partial \Gamma_{\alpha}=\gamma_{\alpha} \cup \gamma_{B \alpha} \cup \gamma_{0}$, $\alpha \in\{\mathrm{L}, \mathrm{R}\}$.
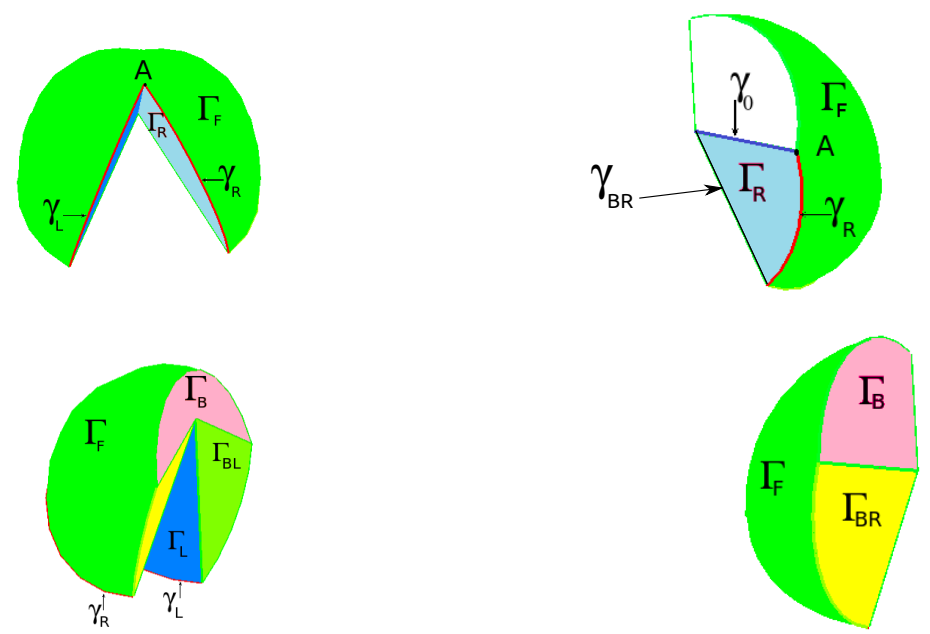

Figure 2. Full breast (left) and half breast (right) in a 3D frontal view (top row) and rear view (bottom row).

\subsection{The functional}

To describe the breast, we use a nonlinear hyperelastic neo-Hookean compressible model as in [17] where we neglect the gravity force assuming that the suturing process is independent of the external field. The problem turns to be the minimization of the associated energy functional $[13,14,15]$ where we determine the new position vector field $f: p \rightarrow f(p)$ which minimizes

$$
\mathcal{J}_{\text {cut }}(f)=\int_{\Omega_{\text {cut }}} W_{\mathrm{br}}(\nabla f(p)) \mathrm{d} p+\int_{\Gamma_{\mathrm{F}}} W_{\mathrm{sk}}\left(\nabla_{\|} f(p)\right) \mathrm{d} S_{p}
$$


subject to the constraints

$$
\begin{array}{ll}
f(p)=p, & \text { in } \Gamma_{\mathrm{B}}, \\
b(f(p))=0, & \text { in } \Gamma_{\mathrm{BL}} \cup \Gamma_{\mathrm{BR}}, \\
\Pi_{\theta}(f(p))=0 & \text { in } \Gamma_{\mathrm{R}} \cup \Gamma_{\mathrm{L}}, \\
f(p)-f(g(p))=0, & \text { in } \gamma_{\mathrm{R}},
\end{array}
$$

where $\Pi_{\theta}$ is the equation of the plane where the suture of the left and right cuts will take place. Indeed, we suppose that for a small asymmetry, i.e. $\alpha_{\mathrm{L}} \approx \alpha_{\mathrm{R}}$, the suturing interface may be approximated by the medium plane. Of course, for large asymmetries, this assumption is no longer valid and the problem of the location of the final interface turns to be more complicated.

The expressions for the volume strain-energy and the skin strain-energy densities are respectively represented by $W_{\mathrm{br}}$ and $W_{\mathrm{sk}}$ and given by

$$
W_{\mathrm{br}}(F)=\frac{\mu_{\mathrm{br}}}{2}\left(\operatorname{tr}\left(F F^{t}\right)-3-2 \ln (\operatorname{det}(F))\right)+\frac{\lambda_{\mathrm{br}}}{2}(\operatorname{det}(F)-1)^{2},
$$

where $F=\nabla f$ is the Jacobi matrix of $f$ and $\left(\lambda_{\mathrm{br}}, \mu_{\mathrm{br}}\right)$ are the Lamé parameters for the breast and

$$
W_{\text {sk }}\left(F_{\|}\right)=\frac{\mu_{\text {sk }}}{2}\left(\operatorname{tr}\left(F_{\|} F_{\|}^{t}\right)-2-2 \ln \left(\operatorname{det}\left(F_{\|}\right)\right)\right)+\frac{\lambda_{\text {sk }}}{2}\left(\operatorname{det}\left(F_{\|}\right)-1\right)^{2},
$$

where $F_{\|}=\nabla_{\|} f$ is the Jacobi matrix of the superficial (skin) displacement $f_{\|}$and $\left(\lambda_{\text {sk }}, \mu_{\text {sk }}\right)$ are the Lamé parameters for the skin. Notice that $f\left(\Omega_{\text {cut }}\right)$ will be the new breast configuration after the suturing that one has to determine.

Condition (2) guarantees that the back interface remains in contact with the trunk while condition (3) states that the rear interfaces belongs to the trunk plane to model the suturing of the breast with the body. To achieve the suturing process with the frontal cut, two boundary conditions are prescribed. The first one (4) states that the junction occurs in a plane $\Pi_{\theta}$ characterized by the angle $\theta$ we shall prescribe as the mean value of the left and right angles while the second condition (5) introduces the mapping $g: \gamma_{\mathrm{R}} \rightarrow \gamma_{\mathrm{L}}$ associated to the suturing. It provides the point-to-point correspondence between the left and right side of the cut, that will be given in section 6 for our specific application.

\section{DISCRETIZATION OF THE SUTURING PROBLEM}

We introduce a discrete version of problem (1)-(5) based on a mesh with tetrahedrons of the breast and a piecewise continuous representation of the new position $f$.

\subsection{Mesh and notations}

We denote by $\mathcal{T}$ a mesh of $\Omega_{\text {cut }}$ constituted of $I$ non-overlapping tetrahedron cells $\tau_{i}, i=$ $1, \ldots, I$, and $N$ vertices $P_{n}=\left(P_{n x}, P_{n y}, P_{n z}\right) \in \mathbb{R}^{3}, n=1, \ldots, N$. Moreover, $T_{k}, k=1, \ldots, K$, represents the faces of the tetrahedrons of the mesh that belong to $\Gamma_{\mathrm{F}}$. Quantities $\left|\tau_{i}\right|$ and $\left|T_{k}\right|$ represent the volume and the area of the cell and the triangle respectively. We also use a local indexation. We denote by $P_{i j}=\left(P_{i j x}, P_{i j y}, P_{i j z}\right) \in \mathbb{R}^{3}, j=1,2,3,4$, the vertices of $\tau_{i}$ and by $P_{k j}=\left(P_{k j x}, P_{k j y}, P_{k j z}\right) \in \mathbb{R}^{3}, j=1,2,3$, the vertices of $T_{k}$. To discretize function $f$, we associate to each node $P_{n}$ an approximation $f_{n} \approx f\left(P_{n}\right)$ and denote by $f_{h}$ the usual continuous piecewise linear function while vector $\Phi_{h}=\left(f_{n x}, f_{n y}, f_{n z}\right)_{p}$ collects the $3 N$ components of the new positions. Vector $\Phi_{h}$ corresponds to the new configuration but the entries are not the unknowns of the problem since some of them are characterized by the boundary or the suturing conditions. Therefore, we extract a sub-vector $X_{h}$ of $\Phi_{h}$ which only contains the unknown values we shall use in the minimisation process while the boundary conditions define an operator

$$
X_{h} \rightarrow \Phi_{h}=\mathcal{B}\left(X_{h}\right)
$$

which provides the other entries to complete vector $\Phi_{h}$. 


\subsection{Boundary and suturing conditions}

Condition (2) yields that $f_{n}=P_{n}$ for any $P_{n}$ on $\Gamma_{\mathrm{B}}$ while relation (3) implies that for any $P_{n}$ on $\Gamma_{\mathrm{BL}} \cup \Gamma_{\mathrm{BR}}$ we set $f_{k y}=0$ to enforce the suturing of the rear cut on the trunk plane $y=0$. To apply Constraint (4), for any $P_{n} \in \Gamma_{\mathrm{R}} \cup \Gamma_{\mathrm{L}}$, we use $f_{n y}$ and $f_{n z}$ as unknowns, i.e. $f_{n y}$ and $f_{n z}$ are entries of $X_{h}$. Therefore, we compute the last component with the relation $f_{n x}=\tan (\theta) f_{n z}$.

Constraint (5) is the main condition since it describes the surgical procedure. For a node $P_{n} \in \gamma_{\mathrm{L}}$, $f_{n y}$ and $f_{n z}$ are entries of $X_{h}$ and we compute the last component with $f_{n x}=\tan (\theta) f_{n z}$ thanks to condition (4). Assume now that function $g: \gamma_{R} \rightarrow \gamma_{L}$ is given (defined in section 6), we proceed in the following way at the discrete level. For a node $P_{n} \in \gamma_{\mathrm{R}}$, we determine the edge $e=\left[P_{\alpha} P_{\beta}\right] \in \gamma_{\mathrm{L}}$ such that $g\left(P_{n}\right) \in e$. We then define the unique barycentric coordinates such that $\lambda_{n \alpha} P_{\alpha}+\lambda_{n \beta} P_{\beta}=g\left(P_{n}\right)$ (see Figure 3).

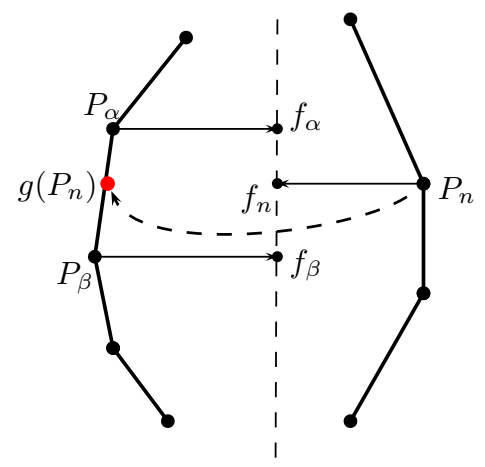

Figure 3. Discretization of function $g$. For any point $P_{n} \in \gamma_{\mathrm{R}}$, we determine an approximation $g\left(P_{n}\right) \in \gamma_{\mathrm{L}}$ between two nodes $P_{\alpha}, P_{\beta}$ of an edge belonging to $\gamma_{\mathrm{L}}$. Barycentric coordinates then derive from the relative position of $g\left(P_{n}\right)$.

To preserve the relative position of $f_{n}$ with respect to $f_{\alpha}$ and $f_{\beta}$ in the final configuration, we set

$$
f_{n}=\lambda_{n \alpha} f_{\alpha}+\lambda_{n \beta} f_{\beta} .
$$

Note that the barycentric coordinates will be computed once as a preprocessing step and none of the coordinates of $P_{n}$ is an entry of $X_{h}$. For any $P_{n}$, the choice of the associated edge $e$ and the barycentric coordinate is equivalent, at the discrete level, to completely define function $g$.

\subsection{Discretization of the functional}

We now introduce a discrete version of the energy functional setting

$$
J_{h}\left(X_{h}\right)=\widehat{J}_{h}\left(\mathcal{B}\left(X_{h}\right)\right)=\widehat{J}_{h}\left(\Phi_{h}\right)=J_{h}^{1}+J_{h}^{2}
$$

constituted of the volume energy $J_{h}^{1}$ and the surface energy $J_{h}^{2}$ we detail hereafter.

3.3.1. Triple integrals of the functional For a new configuration characterized by the approximation $p \in \Omega_{\text {cut }} \rightarrow f_{h}(p) \in \mathbb{R}^{3}$ and stored in vector $\Phi_{h}$, the internal energy on tetrahedron $\tau_{i}$ is given by

$$
W_{\tau_{i}}=\left|\tau_{i}\right|\left(\frac{\mu}{2}\left[\operatorname{tr}\left(F_{i} F_{i}^{t}\right)-3-2 \ln \left(\operatorname{det}\left(F_{i}\right)\right)\right]+\frac{\lambda}{2}\left[\operatorname{det}\left(F_{i}\right)-1\right]^{2}\right),
$$

where $F_{i}$ is the $3 \times 3$ matrix solution of the linear system

$$
f_{i 2}-f_{i 1}=F_{i}\left(P_{i 2}-P_{i 1}\right), \quad f_{i 3}-f_{i 1}=F_{i}\left(P_{i 3}-P_{i 1}\right), \quad f_{i 4}-f_{i 1}=F_{i}\left(P_{i 4}-P_{i 1}\right) .
$$

The total internal energy is then approximated by $J_{1}^{h}=\sum_{\tau_{i} \in \Omega_{\mathrm{cut}}} W_{\tau_{i}}$. 
3.3.2. Surface integrals of the functional The discrete piecewise linear function $f_{h}$ transforms a triangle $T_{k}$ with vertices $O A B$ into a triangle $T_{k}^{\prime}$ with vertices $O^{\prime} A^{\prime} B^{\prime}$. Since the translation and the rotation do not change the stress due to the deformation, we assume that $O^{\prime} B^{\prime}$ is collinear to $O B$ and $A^{\prime}$ belongs to the same plane than triangle $O A B$. Function $f_{\|}$is a two-dimensional function locally given by $f_{\|}(O)=O, f_{\|}(A)=A^{\prime}, f_{\|}(B)=B^{\prime}$. The Jacobian matrix of $f_{\|}$is the constant matrix

$$
J f_{\|}=\mathcal{A}=\left[\begin{array}{ll}
a & b \\
c & d
\end{array}\right]
$$

To determine the matrix, one writes

$$
\left[\begin{array}{ll}
a & b \\
c & d
\end{array}\right]\left[\begin{array}{c}
\|O B\| \\
0
\end{array}\right]=\left[\begin{array}{c}
\left\|O^{\prime} B^{\prime}\right\| \\
0
\end{array}\right], \quad\left[\begin{array}{ll}
a & b \\
c & d
\end{array}\right]\left[\begin{array}{c}
\|O A\| \cos (\alpha) \\
\|O A\| \sin (\alpha)
\end{array}\right]=\left[\begin{array}{c}
\left\|O^{\prime} A^{\prime}\right\| \cos \left(\alpha^{\prime}\right) \\
\left\|O^{\prime} A^{\prime}\right\| \sin \left(\alpha^{\prime}\right)
\end{array}\right]
$$

where $\alpha=\angle(O A, O B)$ and $\alpha^{\prime}=\angle\left(O^{\prime} A^{\prime}, O^{\prime} B^{\prime}\right)$. The first linear system gives $c=0$ and $a=$ $\frac{\left\|O^{\prime} B^{\prime}\right\|}{\|O B\|}$. Substituting these expressions in the second linear system we obtain

$$
d=\frac{\left\|O^{\prime} A^{\prime}\right\| \sin \left(\alpha^{\prime}\right)}{\|O A\| \sin (\alpha)}, \quad c=\frac{\left\|O^{\prime} A^{\prime}\right\| \cos \left(\alpha^{\prime}\right)-a\|O A\| \cos (\alpha)}{\|O A\| \sin (\alpha)}
$$

The superficial energy on triangle $T$ for the skin is then given by

$$
W_{T}=|T|\left(\frac{\mu_{\mathrm{sk}}}{2}\left[\operatorname{tr}\left(J f_{\|} J f_{\|}^{t}\right)-3-2 \ln \left(\operatorname{det}\left(J f_{\|}\right)\right)\right]+\frac{\lambda_{\mathrm{sk}}}{2}\left[\operatorname{det}\left(J f_{\|}\right)-1\right]^{2}\right)
$$

and the whole superficial energy is approximated by

$$
J_{h}^{2}=\sum_{T \in \Gamma_{F}} W_{T} .
$$

\subsection{Minimization algorithm}

For a given vector $X_{h}$ and the boundary conditions, we deduce vector $\Phi_{h}=\mathcal{B}\left(X_{h}\right)$, hence the continuous linear piecewise function $f_{h}$ we use to compute the discretize energy functional. In order to determine the minimizer $\bar{X}_{h}$ of the discrete functional $J_{h}\left(X_{h}\right)$, we employ the conjugate gradients method. To this end, one has to compute an approximation of the derivative of the discrete functional in directions $\ell=1, \ldots, \# X_{h}$. Since the function is nonlinear and has complex structure, numerical derivatives are computed in a very simple way. For example, an approximation of derivative with respect to direction $\ell$ at point $X$ is given by

$$
\partial_{\ell} J_{h}(X) \approx \frac{J_{h}\left(X+\epsilon_{\ell}\right)-J_{h}(X)}{\epsilon},
$$

where $\epsilon_{\ell}$ is a vector of zeros except value $\epsilon$ for the $\ell$-th entry.

\section{BREAST UNDER GRAVITATIONAL FIELD}

After the suturing, the two faces $\Gamma_{\mathrm{L}}$ and $\Gamma_{\mathrm{R}}$ are in contact and the question arises of the breast shape in the gravitational field in function of the trunk position. Two new contributions are now considered for the energy function: the gravity force and the introduction of the Chassaignac space. The last item corresponds to a mobile zone located between the breast and the trunk which acts as a spring to maintain the breast close to the trunk. The initial geometry $\Omega_{\text {gra }}$ we shall deal with is the breast after the suturing process where the two interfaces $\Gamma_{\mathrm{L}}$ and $\Gamma_{\mathrm{R}}$ are in contact. To determine the new shape, we assume that surfaces are stick together in such a way that the relative position of the nodes of $\Gamma_{R}$ (resp. $\gamma_{R}$ ) with respect to the nodes of $\Gamma_{L}$ (resp. $\gamma_{L}$ ) will be preserved when the gravity field is presented. Notice that no new meshing have been performed hence the two areas are still considered 

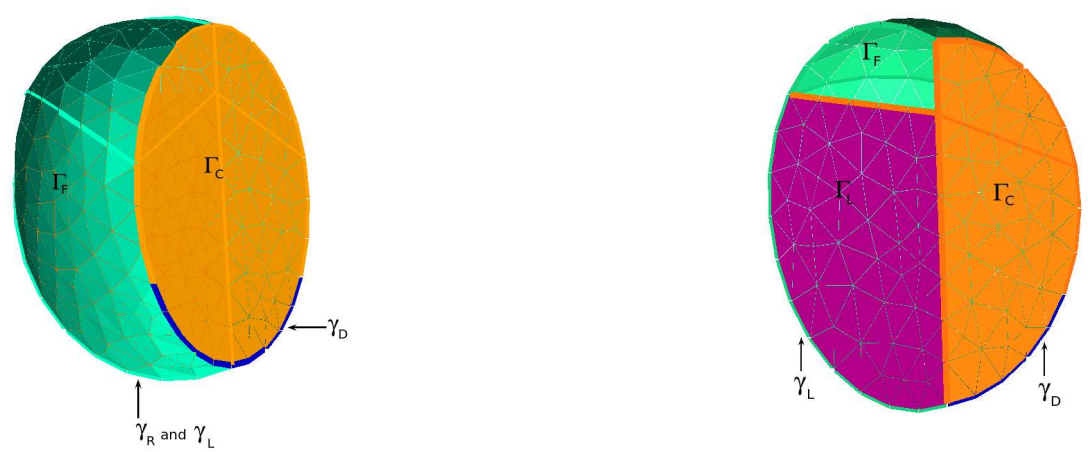

Figure 4. 3D rear views for the gravity problem: full breast (left) and half breast (right).

as distinct parts of the boundary with a non-matching mesh. Therefore a specific condition, named the stick condition, is added to maintain the two faces in contact.

Figure 4 displays the breast geometry domain $\Omega_{\text {grav }}$ deriving from the suturing process and we introduce two new ingredients for the modelling. A new curve $\gamma_{D}$ on the infra-mammary fold is defined where the breast is fixed. We also merge the interfaces $\Gamma_{B}, \Gamma_{B L}$ and $\Gamma_{B R}$ into a unique interface $\Gamma_{C}$ where the Chassaignac condition is prescribed. The problem turns to the minimization of the associated energy functional where we determine the new position vector field $f: p \rightarrow f(p)$ which minimizes

$$
\begin{array}{r}
\mathcal{J}_{\text {gra }}(f)=\int_{\Omega_{\text {gra }}} W_{\text {br }}(\nabla f(p)) \mathrm{d} p+\int_{\Gamma_{\mathrm{F}}} W_{\text {sk }}\left(\nabla_{\|} f(p)\right) \mathrm{d} S_{p}- \\
\int_{\Omega_{\text {gra }}} \rho a_{\mathrm{g}} \cdot f(p) \mathrm{d} p+\int_{\Gamma_{\mathrm{C}}} c\|f(p)-p\| \mathrm{d} S_{p}
\end{array}
$$

subject to the constraints

$$
\begin{array}{ll}
f(p)=p, & \text { in } \gamma_{\mathrm{D}}, \\
h(f(p))=0, & \text { in } \Gamma_{\mathrm{C}}, \\
f(p)-f(G(p))=0, & \text { in } \Gamma_{\mathrm{R}} \cup \gamma_{\mathrm{R}},
\end{array}
$$

where $a_{g}$ is the gravity acceleration, $c$ the Chassaignac coefficient and $\rho$ the density.

Condition (7) corresponds to fix the breast displacement on curve $\gamma_{\mathrm{D}}$ while condition (8) maintains the breast on the trunk. Condition (9) is the stick condition that preserves the junction between the left and right interfaces previously joined in the suturing process. Function $G$ results from the suturing process which defined the mapping from $\Gamma_{L}$ (resp. $\gamma_{L}$ ) onto $\Gamma_{R}$ (resp. $\gamma_{R}$ ) and will be given in section 5.1 .

\section{DISCRETIZATION OF THE BREAST UNDER THE GRAVITATIONAL FIELD}

We introduce a discrete version of problem (6)-(9) based on a mesh of tetrahedrons of the breast and a continuous piecewise representation of the displacement $f$. Due to the new boundary and constraint conditions, we have to deal with a new subset of unknowns $Y_{h}$ from $\Phi_{h}$ and a new operator $\mathcal{C}$ such that $Y_{h} \rightarrow \Phi_{h}=\mathcal{C}\left(Y_{h}\right)$ models all the constraints (7)-(9).

\subsection{Boundary, Chassaignac and sticking conditions}

Condition (7) yields that $f_{n}=P_{n}$ for any $P_{n} \in \gamma_{D}$ while relation (8) implies that for any $P_{n} \in \Gamma_{C}$, we set $f_{n y}=0$ to maintain interface $\Gamma_{C}$ on the trunk plane $y=0$. Constraint (9) means that the two interfaces previously joined and stick will preserve their relative position and we define function 
$G$ in the following way. At the initial stage, after reading the mesh, we compute the barycentric coordinates for points $P_{n}$ on $\gamma_{R}$ and $\Gamma_{R}$ corresponding to the suturing process:

- If $P_{n} \in \gamma_{R}$, we determine the edge $e=\left[P_{\alpha}, P_{\beta}\right] \subset \gamma_{L}$ such that $P_{n}$ also belongs to $e$ since the two curves are joined. We then define the unique barycentric coordinates such that $G\left(P_{n}\right)=\lambda_{n a} P_{\alpha}+\lambda_{n \beta} P_{\beta}=P_{n}$. Hence the constraint on the new location $f_{n}$ associated to point $P_{n}$ writes $f_{n}=\lambda_{n a} f_{\alpha}+\lambda_{n \beta} f_{\beta}$ where $f_{\alpha}$ and $f_{\beta}$ are the new positions of $P_{\alpha}$ and $P_{\beta}$, respectively. Notice that $f_{n}$ does not belongs to the entries of $Y_{h}$ since we deduce it from the linear combination of $P_{\alpha}, P_{\beta}$.

- If $P_{n} \in \Gamma_{R}$, we determine the triangle $f=\left[P_{i} P_{j} P_{k}\right] \subset \Gamma_{L}$ such that $P_{n}$ also belongs to $f$ since the two surfaces are sutured. We then define the unique barycentric coordinates such that $G\left(P_{n}\right)=\lambda_{n i} P_{i}+\lambda_{n j} P_{j}+\lambda_{n k} P_{k}=P_{n}$. Hence the constraint on the new location $f_{n}$ associated to point $P_{n}$ writes $f_{n}=\lambda_{n i} f_{i}+\lambda_{n j} f_{j}+\lambda_{n k} f_{k}$ where $f_{i}, f_{j}$ and $f_{k}$ are the new positions of $P_{i}, P_{j}$ and $P_{k}$, respectively. Notice once that $f_{n}$ does not belongs to the entries of $Y_{h}$ since we deduce it from the linear combination of $P_{i}, P_{j}, P_{k}$.

\subsection{Discretisation of the functional}

We introduce a discrete version of the energy functional setting

$$
J_{h}\left(Y_{h}\right)=\widetilde{J}_{h}\left(\mathcal{C}\left(Y_{h}\right)\right)=\widetilde{J}_{h}\left(\Phi_{h}\right)=J_{h}^{1}+J_{h}^{2}+J_{h}^{3}+J_{h}^{4}
$$

with

$$
J_{h}^{3}=-\sum_{\tau_{i}} \frac{\left|\tau_{i}\right|}{4} \rho a_{g}\left(f\left(P_{i 1}\right)+f\left(P_{i 2}\right)+f\left(P_{i 3}\right)+f\left(P_{i 4}\right)\right)
$$

and

$$
\left.J_{h}^{4}=\sum_{T_{j} \subset \Gamma_{C}} \frac{\left|T_{j}\right|}{3} c\left(\left\|f\left(P_{i 1}\right)-P_{i 1}\right\|+\left\|f\left(P_{i 2}\right)-P_{i 2}\right\|+\left\|f\left(P_{i 3}-\right) P_{i 3}\right\|\right)\right) .
$$

Minimization of functional $J_{h}$ is performed in the similar way as presented in section 3.4.

\section{NUMERICAL SIMULATIONS}

The original breast is modelled as a truncated sphere characterized by the its principal radius and the length of the truncated portion. Figure 5 displays the initial configuration of the breast, i.e. before the incisions. We would like to highlight that more complex geometries can easily be considered and the suturing method is not limited to that particular configuration.
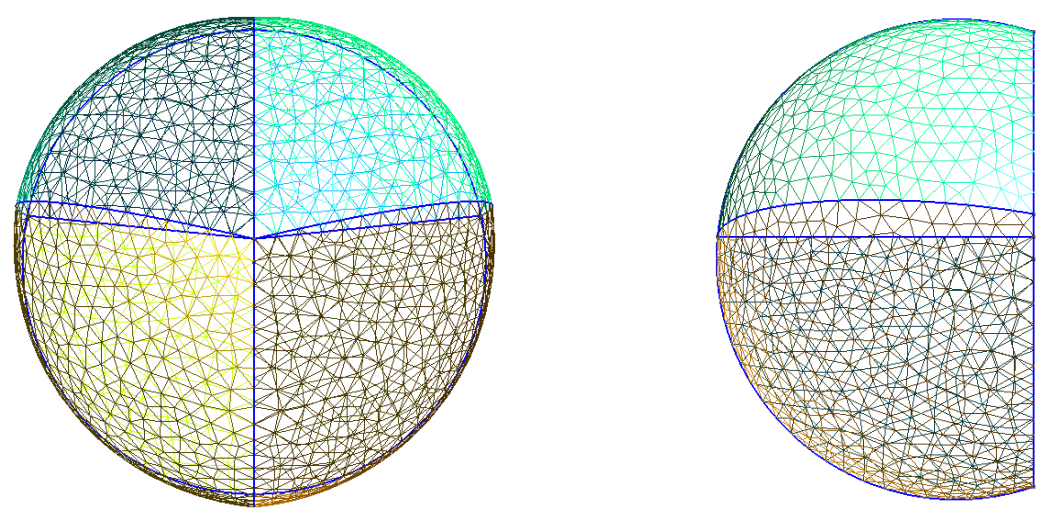

Figure 5. Mesh of the pre-operative breast: frontal view (left) and lateral view (right). 


\subsection{The suturing mapping $g$}

Function $g$ is the fundamental information to describe the suturing process by defining the mapping from $\gamma_{\mathrm{R}}$ onto $\gamma_{\mathrm{L}}$ which physically represents the skin junction performed by the surgeon. To this end, we propose a method to provide such a mapping based on the principle of equivalent relative distance. More precisely, since $A$ is a fixed point, for any $P \in \gamma_{\mathrm{R}}$, we compute the distance of arc $\widehat{A P}$ denoted by $|\widehat{A P}|$ and define the relative distance

$$
r(P)=\frac{\widehat{A P} \mid}{\left|\gamma_{\mathrm{R}}\right|}
$$

where $\left|\gamma_{\mathrm{R}}\right|$ is the length of the curve $\gamma_{\mathrm{R}}$. We then take the associated $g(P)$ such that $|\widehat{\operatorname{Ag}(P)}|=$ $r(P)\left|\gamma_{\mathrm{L}}\right|$. At the numerical level, for a node $P_{n} \in \gamma_{\mathrm{R}}$, we seek the edge $e=\left[v_{\ell 1}, v_{\ell 2}\right] \subset \gamma_{L}$ such that $g\left(P_{n}\right) \in e$ and compute the barycentric coordinates by solving equation

$$
g\left(P_{n}\right)=\lambda_{1} v_{\ell 1}+\lambda_{2} v_{\ell 2}, \quad \lambda_{1}+\lambda_{2}=1 .
$$

We store in a vector the correspondence between vertices and edges and the associated barycentric coefficients.

\subsection{Symmetric suturing}

In the first numerical test, we performed a symmetric frontal incision with $\alpha_{\mathrm{L}}=\alpha_{\mathrm{R}}=30^{\circ}$ with no rear cut. Figure 6 gives two views of the configuration before the suturing while we display in Figure 7 the final configuration after the suturing. We have chosen $\theta=0^{\circ}$ to preserve the symmetry of the junction.
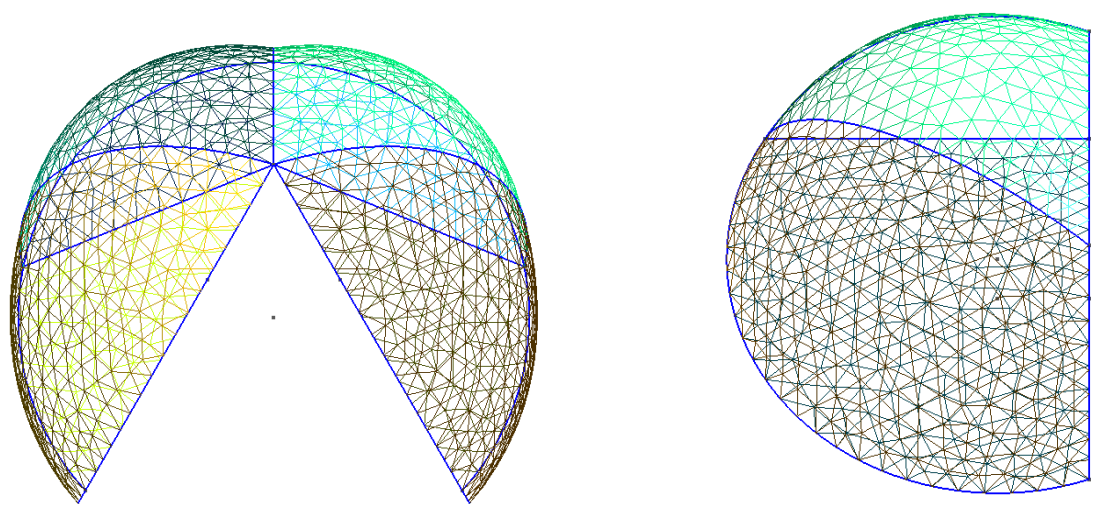

Figure 6. Mesh of the breast after a symmetric incision: frontal view (left) and lateral view (right).

We notice that the symmetry of the shape is preserved and we have check the convergence to a limit configuration using finer meshes.

We then evaluate the breast shape subject to the gravity field for a person in stand up position. Figure 8 displays the frontal and side view of the breast under the gravity field and we notice that the symmetry is also preserved and the junction obtained is a straight line. Computations have been carried out with finer meshes to check that the solution converges to a limit one. The presented solution involves a mesh of 1246 tetrahedra and is very closed to the one obtain with finer meshes (less that $1 \%$ of relative errors).

\subsection{Asymmetric suturing}

In some situations, the surgeon has to perform asymmetric incisions and we experiment other code to assess the its capacity to handle such a more complex case. Figure 9 presents the initial breast 

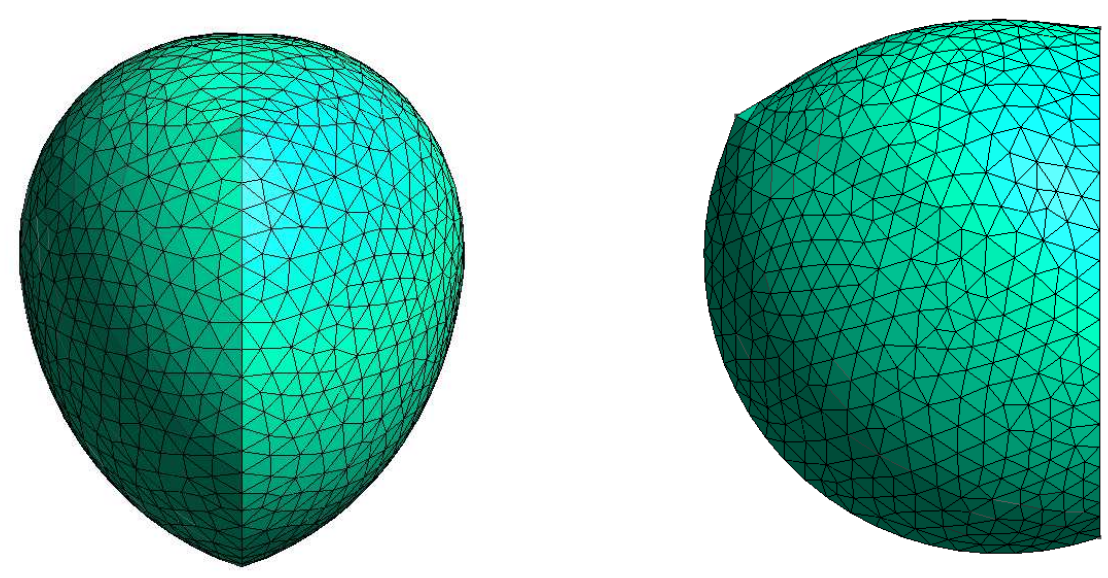

Figure 7. Shape of the breast after the suturing without gravity field: frontal view (left) and lateral view (right).
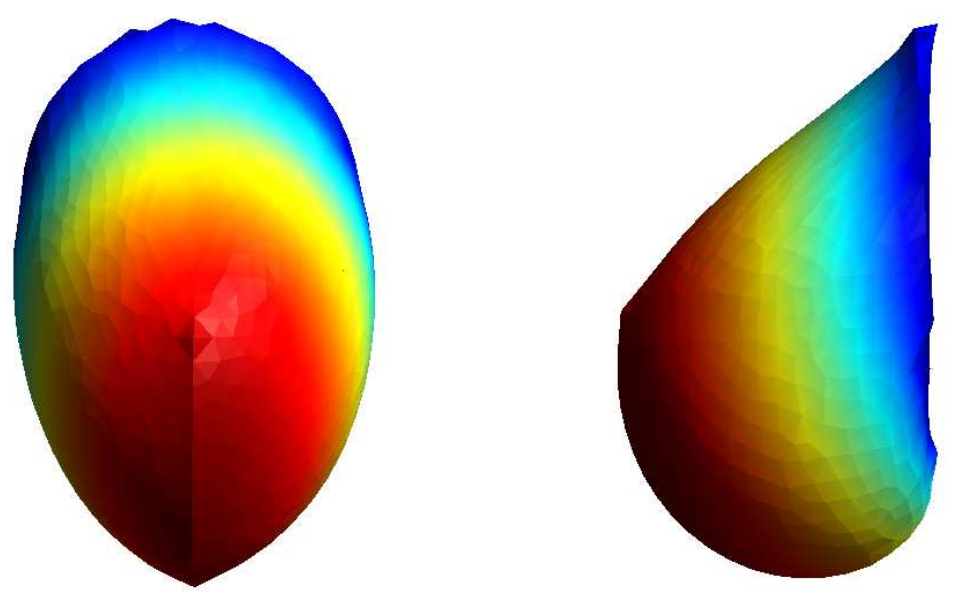

Figure 8. The breast under the gravity field: frontal view (left) and lateral view (right).

before the suturing where we have choose $\alpha_{L}=15^{\circ}$ and $\alpha_{R}=45^{\circ}$. Clearly the breast volume of the right side is strongly reduced in comparison with the left side and the junction plane is characterized with $\theta=0^{\circ}$.

Computations are carried out with a 1376 tetrahedra mesh taking the angle $\theta_{0}=(-15+45) / 2$ of the suturing plan as the (algebraic) mean values of the two angles. Figure 10 plots the shape of the breast after the suturing process and the asymmetry is noticeable. As in the former case, we obtained shape convergence using finer meshes which guarantee the stability of the method.

We perform the computation of the suturing breast under the gravity field and we report that the suture location is no longer a straight line as displayed in Figure 11. Convergence to a limit configuration has been checked using finer meshes.

\subsection{Frontal and rear suturing}

As a final test, we consider a more complex case where we perform both a front and a rear incision of the breast. Figure 12 shows the mesh corresponding to two incisions while Figure 13 prints the configuration after the numerical suturing process. 

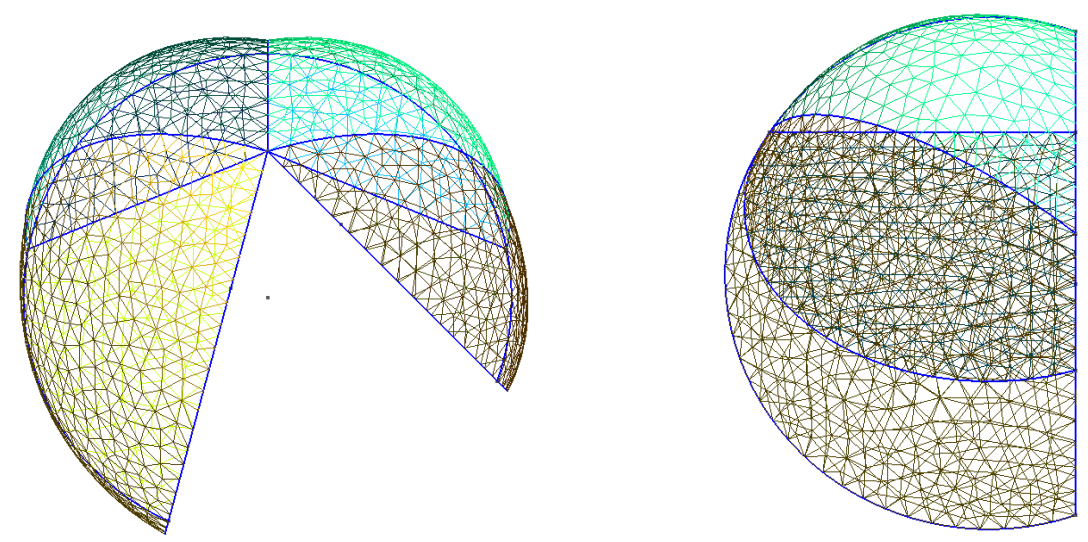

Figure 9. Mesh of the breast after an asymmetric incision: frontal view (left) and lateral view (right)..
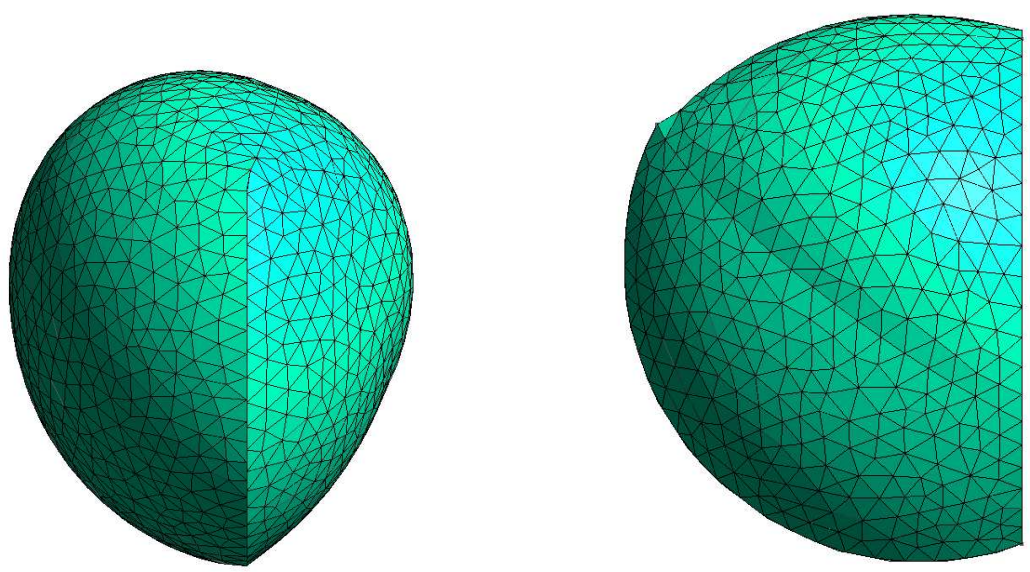

Figure 10. Mesh of the breast after suturing: frontal view (left) and lateral view (right).
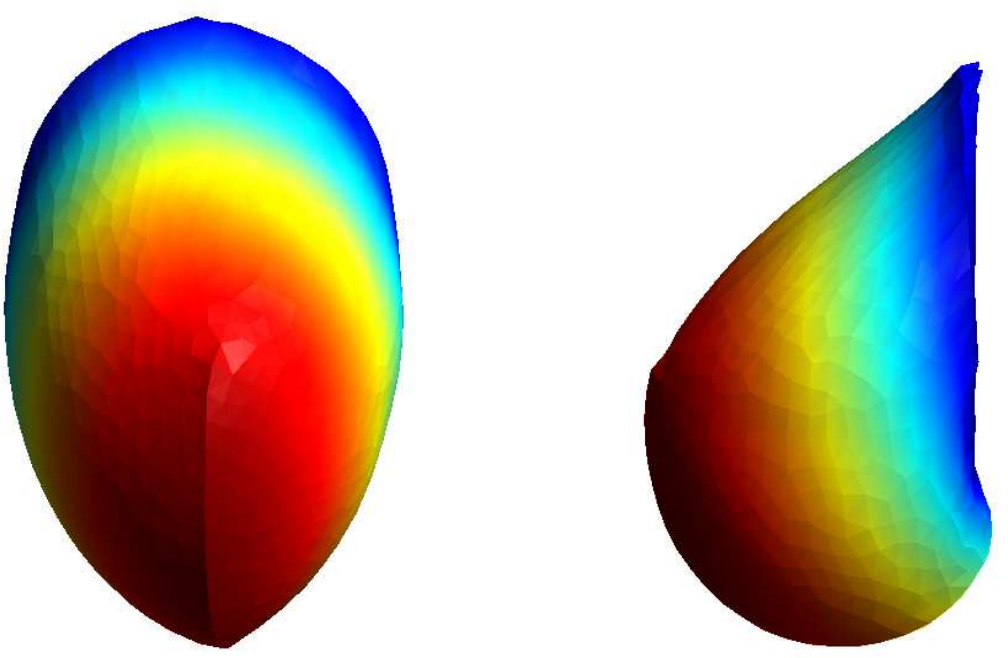

Figure 11. The breast under the gravity field: frontal view (left) and lateral view (right). 

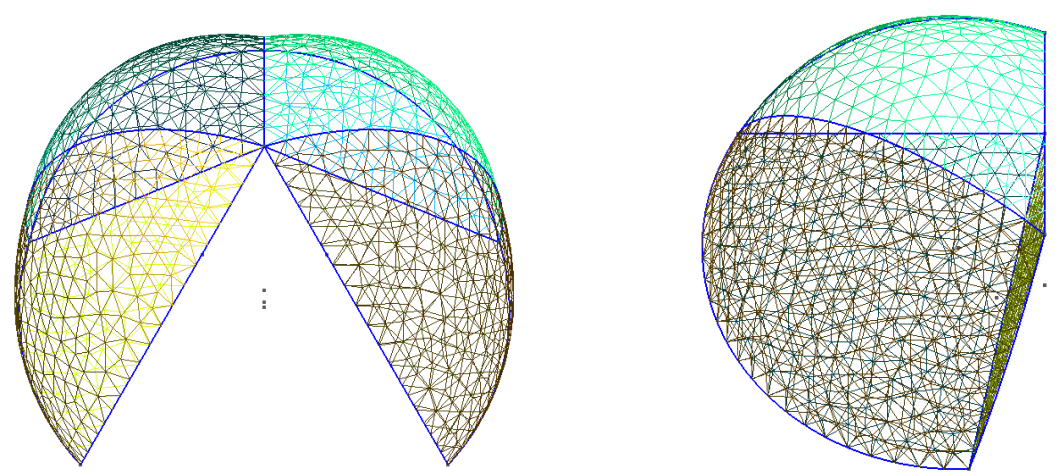

Figure 12. Mesh of the breast after a symmetric front incision and a rear incision: frontal view (left) and lateral view (right).

We use a 1539 tetrahedra mesh presented in Figure 13 but have experimented several finer meshes to check the convergence. No significance differences (lower than 1\%) have been reported with respect to the solution.
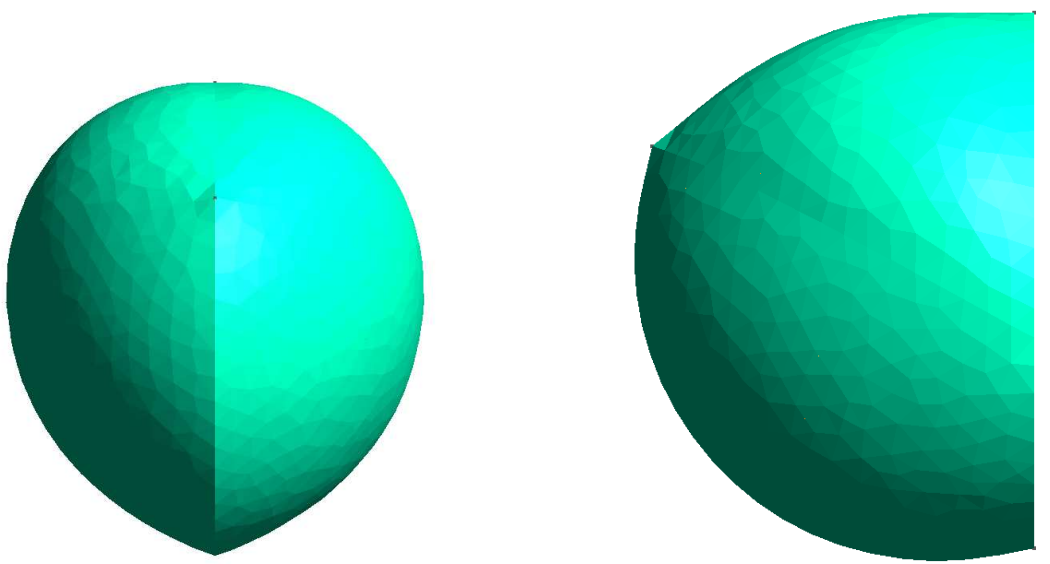

Figure 13. Breast shape after the suturing process: frontal view (left) and lateral view (right).

We also present in Figure 14 the picture corresponding to the deformation of the breast when applying the gravity field. Such a final snapshot will represent the expected shape of the breast after the operation.

\section{CONCLUSION}

The software developed for breast reduction modelling allows one to forecast the final breast geometry according to the incision marking parameters. The main point is to perform symmetric or non-symmetric suturing between two interfaces with non-matching meshes while preserving the final junction when we apply the gravitational field. Accuracy of the approximation with rough meshes is sufficient for a satisfactory analysis of errors frequently done during breast reduction surgery and allows to understand how to avoid or correct them. The next step will be the comparison of the simulations with real surgery cases to validate our approach from a practical point of view. 

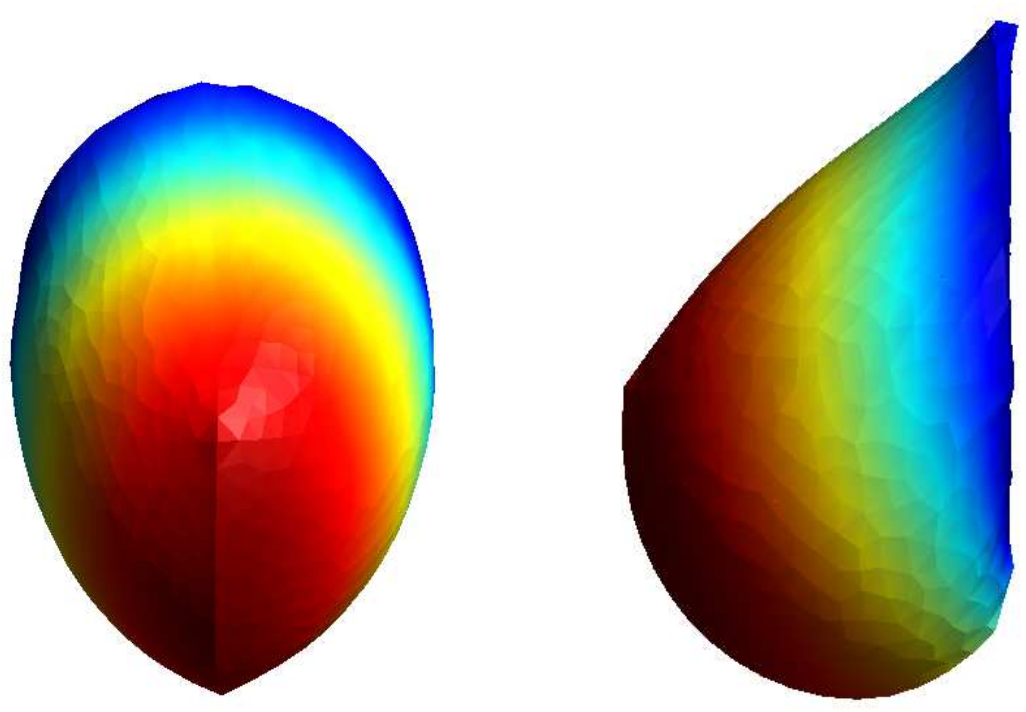

Figure 14. The suturing breast in the gravity field: frontal view (left) and lateral view (right).

\section{ACKNOWLEDGEMENTS}

The authors are grateful to Professor Augusta Cardoso and Professor Horácio Costa. This work was supported with the Portuguese Funds from the "Fundação para a Ciência e a Tecnologia" through the Project EXPL/MAT-NAN/0606/2013. The authors were also supported by the Research Centre of Mathematics and the Research Centre of Physics of the University of Minho with the Portuguese Funds from the "Fundação para a Ciência e a Tecnologia", through the Project PEstOE/MAT/UI0013/2014 and PEst-C/FIS/UI0607/2013.

\section{REFERENCES}

1. Balaniuk R, Costa I, Melo J. Cosmetic Breast Surgery Simulation. Annals of the VIII Symposium on Virtual Reality 2006; 387-396.

2. Cardoso A, Coelho G, Zenha H, Sá V, Smirnov G, Costa H. Computer Simulation of Breast Reduction Surgery. Aesth. Plast. Surg. 2003; 37:68-76.

3. Azar FS, Metaxas DX, Schnall MD. Methods for modeling and predicting mechanical deformations of the breast under external perturbations. Medical Image Analysis 2002; 6:1-27.

4. Ayache N, Delingette H. Surgery Simulation and Soft Tissue Modeling. Lecture Notes in Computing Science 2003; Springer, Berlin.

5. Perez-del-Palomar A, Calvo B, Herrero J, López J, Doblaré M. A finite element model to accurately predict real deformations of the breast. Medical Engineering \& Physics 2008; 30:1089-1097.

6. Delingette H. Towards realistic soft tissue modeling in medical simulation. Proceedings of IEEE: Special Issues on Surgery Simulation April 1998; 512-523.

7. Hernádez-Gascón B, Espés N, Peña E, Pascual G, Bellón JM, Calvo B. Computational framework to model and design surgical meshes for hernia repair. Computer Methods in Biomechanics and Biomedical Engineering 2014; 17:1071-1085.

8. Fung YC. Biomechanics - Mechanical Properties of Living Tissues. Springer-Verlag, 2nd edition, 2012.

9. Rajagopal V, Nielsen PMF, Nash MP. Development of a Three-Dimensional Finite Element Model of Breast Mechanics. IEEE-EMBS 26th Ann. Intl. Conf., San Francisco 2004; 5080-5083.

10. Payan Y (Ed.). Soft Tissue Biomechanical Modeling for Computer Assisted Surgery. Springer, Heidelberg, 2012.

11. Zhong H, Wachowiak MP, Peters TM. A real time finite element based tissue simulation method incorporating nonlinear elastic behavior. Computer Methods in Biomechanics and Biomedical Engineering 2005; 8:177-189.

12. Lee HP, Zhuang H. Biomechanical study on the edge shapes for penetrating keratoplasty. Computer Methods in Biomechanics and Biomedical Engineering 2012; 15:1071-1079

13. Ball JM. Energy-Minimizing Configurations in Nonlinear Elasticity. Proceedings of the International Cogress of Mathematicians, Warzawa August 1983.

14. Ciarlet P. Three-Dimensional Elasticity, Elsevier, Amsterdam, 1998.

15. Picinbono G, Delingette H, Ayache N. Non-linear anisotropic elasticity for real-time surgery simulation. Graphical Models 2003; 305-321. 
14 D. LOPES, S. CLAIN, R.M.S. PEREIRA, G.J. MACHADO, G. SMIRNOV, I. VASILEVSKIY, H. COSTA

16. Clain S, Machado GJ, Pereira RMS, Smirnov G. Soft tissue modelling for analysis of errors in breast reduction surgery. 11th World Congress on Computational Mechanics - WCCM XI, Barcelona July 2014; 1678-1687.

17. Cardoso A, Costa H, Sá V, Smirnov G. On the importance of Chassaignac's space in breast modelling. IV European Conference on Computational Mechanics, Paris, May 2010; 16-21. 